\title{
Open questions: Missing pieces from the immunological jigsaw puzzle
}

\author{
Gillian M Griffiths*
}

There is nothing more frustrating than reaching the end of a jigsaw puzzle to find that some of the pieces are missing. There are certainly areas of immunology where the same problem applies and despite major advances there are some pieces of the jigsaw that are still missing after many years. When I was asked to think of some of the questions that remain unanswered in the cell biology of the immune system, three leapt to mind. One concerns antigen presentation, another cytotoxic $\mathrm{T}$ lymphocyte (CTL) - or natural killer (NK)-cell-mediated killing, and the third a mechanism of apoptosis. Although seemingly disparate, each one of these boils down to a question of how proteins cross membranes to reach the cytoplasm.

\section{The missing step that allows 'cross-presentation'}

The first is the mechanism that lies at the heart of 'crosspresentation' [1] and allows extracellular antigens to be endocytosed by dendritic cells, released into the cytoplasm, and displayed to cytotoxic $\mathrm{T}$ lymphocytes through the proteasomal/MHC class I pathway that operates in the presentation of intracellular antigens (Figure 1). Although it has been clear for many years that proteins are released from endocytic compartments into the cytoplasm [2], where they are exposed to the proteasomal pathway, the mechanism and trigger for this critical transport step remain unclear.

\section{How do cytotoxic cells avoid self-destruction?}

The second problem, which remains much debated over many years, concerns how and where the pore-forming protein perforin, used by CTL and NK cells to destroy their targets, does or does not cross membranes. A great deal of research has focused on whether perforin forms

*Correspondence: gg305@cam.ac.uk

Cambridge Institute for Medical Research, Hills Rd, Cambridge, CB2 OXY, UK

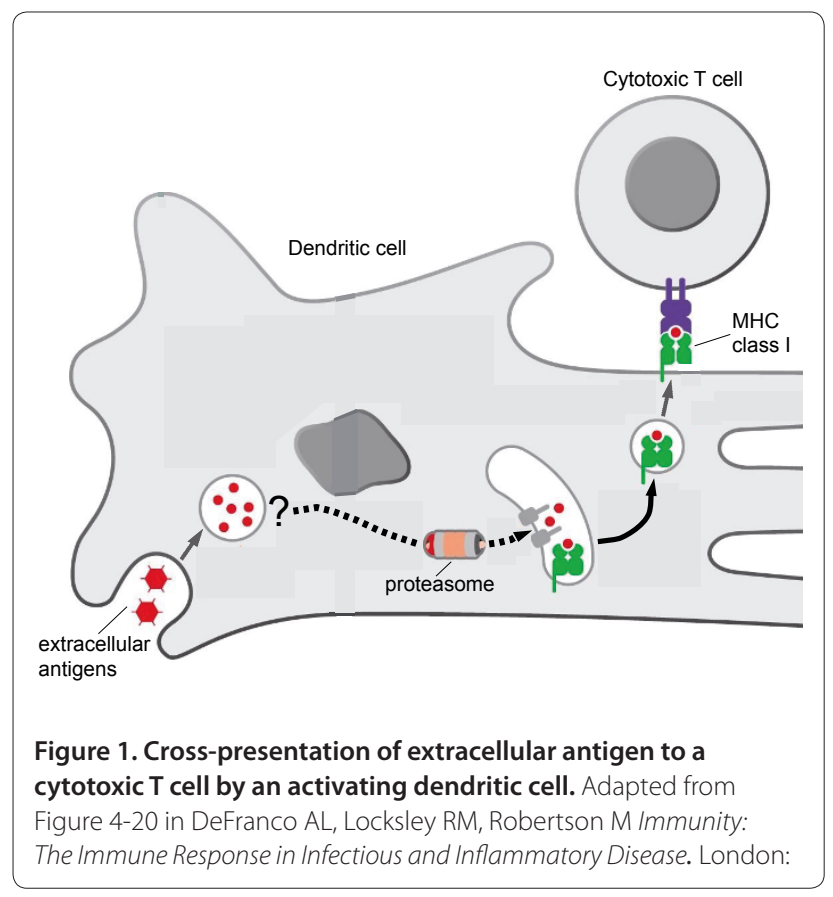

its pore as it reaches the plasma membrane or is first taken up into endosomes. However a knottier and much less studied problem is how CTLs resist killing by perforin as they kill their targets. Why is the integrity of the CTL plasma membrane not compromised during target cell killing? There is no innate mechanism of resistance in the plasma membrane, since CTLs can themselves be targets. To date the only mechanism proposed for this resistance is a protective coating of cathepsin B (capable of cleaving perforin and rendering it non-functional) on the inside of the secretory granules 


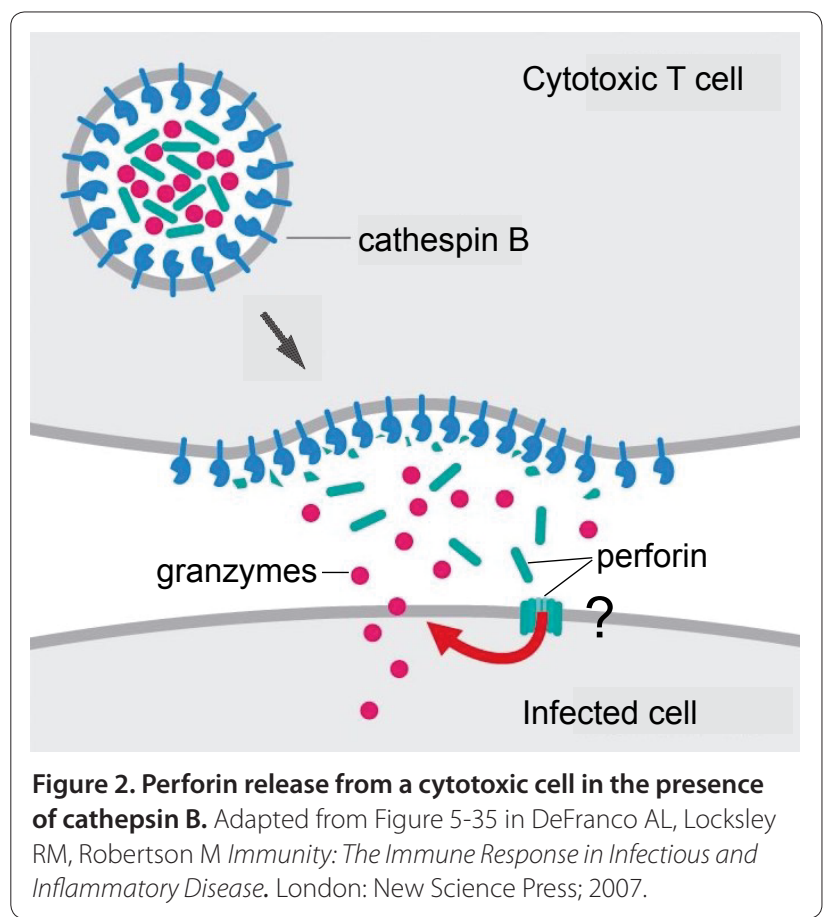

from which perforin is released [3] (Figure 2). However, since cathepsin B-deficient CTL do not self-destruct upon killing [4], it is clear that this cannot be the only protective mechanism in play.

\section{A chicken-and-egg problem in apoptosis}

The third problem concerns apoptosis. One recently proposed pathway of caspase-independent apoptosis [5] suggests that the initial event triggering cell death is rupture of the lysosomal membranes releasing cathepsins that can be active in the cytoplasm. This pathway is thought to provide an additional mechanism for triggering apoptosis. There is something of a chickenand-egg problem, however, as it is difficult to be sure that lysosomal breakdown is the initiating event rather than a consequence of rapid cell death. However, the key question that remains is how the membrane is ruptured to allow the proteins to escape into the cytoplasm.

Published: 31 January 2013

\section{References}

1. Joffre OP, Segura E, Savina A, Amigorena S. Cross-presentation by dendritic cells. Nat Rev Imm 2012, 12:557-569.

2. Norbury CC, Hewlett LJ, Prescott AR, Shastri N, Watts C: Class I MHC presentation of exogenous soluble antigen via macropinocytosis in bone marrow macrophages. Immunity 1995, 3:783-791.

3. Balaji KN, Schaske N, Machleidt W, Catalfamo M, Henkart PA: Surface cathepsin B protects cytotoxic lymphocytes from self-destruction after degranulation. J Exp Med 2002, 196:493-503.

4. Baran K, Ciccone A, Yagita H, Bird PI, Villadangos JA, Trapani JA: Cytotoxic T lymphocytes from cathepsin B-deficient mice survive normally in vitro and in vivo after encountering and killing target cells. J Bio/ Chem 2006, 281:30485-30491.

5. Jäättelä M, Tschopp J: Caspase-independent cell death in T lymphocytes. Nat Immunol 2003, 4:416-423.

doi:10.1186/1741-7007-11-10

Cite this article as: Griffiths GM: Open questions: Missing pieces from the immunological jigsaw puzzle. BMC Biology 2013, 11:10. 